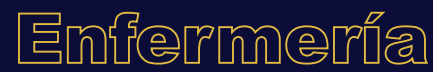

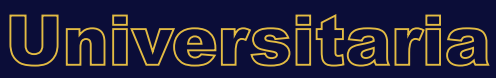

\section{Experiencia de violencia laboral en profesionales de enfermería de un hospital público}

\section{Experience of workplace violence against nursing professionals of a public hospital}

\section{Experiência de violência no trabalho em profissionais de enfermagem de um hospital público}

\section{S. Cerda-Antilef ${ }^{a 1}$, E. Rivas-Riveros ${ }^{b 2^{*}}$, M. Campillay-Campillay ${ }^{\mathrm{c}}$}

ORCID

a oooo-0001-9666-153X

${ }^{\mathrm{c} 0000-0002-4054-1595}$

${ }^{\mathrm{b}} \mathrm{0} 000-0002-9832-4534$

${ }^{1}$ Universidad San Sebastián, Facultad de Medicina, Departamento de Enfermería, Valdivia, Chile

${ }^{2}$ Universidad de La Frontera, Facultad de Medicina, Departamento de Enfermería, Temuco, Chile

3 Universidad de Atacama, Facultad de Ciencias de la Salud, Departamento de Enfermería, Copiapó, Chile

Recibido: 27 enero 2020

Aceptado: 28 septiembre 2020

\section{RESUMEN}

Introducción: La violencia laboral en el entorno clínico es un tema emergente y de gran relevancia para los profesionales de enfermería, ya que afecta la eficiencia de los cuidados, y daña la salud de los profesionales que la sufren. A nivel global se ha evidenciado mayor violencia en entornos feminizados.

Objetivo: Comprender la experiencia de violencia laboral entre profesionales de enfermería hospitalarios durante el año 2017 en la ciudad de Valdivia, Chile. 
Método: La investigación se aborda desde el paradigma interpretativo, metodología cualitativa y análisis de contenido de acuerdo a lo propuesto por Bardin. La muestra fue intencionada y se realizó entrevista en profundidad a diez profesionales de enfermería que habían experimentado violencia laboral y consintieron su participación. Se aplicaron preguntas orientadoras para conocer barreras y facilitadores de violencia.

Resultados: Emergen cuatro categorías: A) Percepción de clima organizacional asociado a estilos de liderazgo; B) Barreras contextuales y actitudinales asociadas a la aparición de conductas violentas (con las subcategorías: falta de colaboración, sobrecarga laboral, falta de procesos y pautas preventivas para la resolución de conflictos); C) Formas de violencia y acoso (con las subcategorías: difundir rumores laborales, persecución, retener información crucial y desautorización); y D) Fatiga de compasión. Se relaciona el fenómeno al modelo biomédico.

Conclusiones: El problema de violencia subyace a determinantes contextuales organizacionales que no dependen exclusivamente de los profesionales de enfermería, por lo que es urgente identificarlas, afrontarlas y prevenirlas.

Palabras clave: Violencia laboral; enfermería; cultura organizacional; agotamiento profesional; Chile.

\section{ABSTRACT}

Introduction: Work related violence in clinic environments has important negative impacts on healthcare professionals and the care they provide. Being a relevant topic, it has been evidenced that the greater work related violence occurs in female prevalent environments. Objective: To gain understanding on the work related violence experienced by nursing professionals in a hospital in the city of Valdivia, Chile, in 2017.

Method: The study follows the interpretative paradigm, using a qualitative methodology, and a content analysis based on the proposals of Bardin. The sample was by-intention. An in-depth interview was conducted on 10 nursing professionals who had experienced work related violence. All participants gave their informed consent. The questions were mainly directed to know barriers and facilitators of violence.

Results: Four categories emerged from the analysis: a) Perception of the organizational climate associated to leadership styles; b) contextual and attitude-related barriers associated to the violent behaviors (with the following subcategories: lack of collaboration, work overload, and lack of processes and preventive measures to conflict resolutions); c) Forms of violence and harassment (with the following subcategories: to create workrelated rumors, prosecution, to retain crucial information, and unauthorization); and d) Compassion fatigue. The phenomenon is related to the biomedical model.

Conclusions: The problem of violence responds to the contextual determinants of the organization, and this factors do not depend exclusively on nursing professionals. Therefore, it is imperative to identify, address, and prevent all work related violence triggers.

Keywords: Workplace violence; nursing; organizational culture; burnout, professional; Chile. 


\section{RESUMO}

Introdução: A violência no local de trabalho no ambiente clínico é um tema emergente e de grande relevância para os profissionais de enfermagem, dado que afeta a eficiência dos cuidados, e prejudica a saúde dos profissionais que a sofrem. A nível global maior violência tem sido evidenciada em ambientes feminizados.

Objetivo: Compreender a experiência de violência no trabalho entre profissionais de enfermagem de hospitais durante o ano 2017 na cidade de Valdivia, Chile.

Método: A pesquisa é abordada desde o paradigma interpretativo, metodologia qualitativa e análise de conteúdo conforme o proposto por Bardin. A amostra foi intencional e realizou-se entrevista em profundidade com dez profissionais de enfermagem que tinham experimentado violência no trabalho e consentiram em sua participação. Aplicaram-se perguntas norteadoras para conhecer barreiras e facilitadores de violência.

Resultados: Emergem quatro categorias: A) Percepção de clima organizacional associado aos estilos de liderança; B) Barreiras contextuais e atitudinais associadas ao aparecimento de comportamentos violentos (com as subcategorias: falta de colaboração, sobrecarga de trabalho, falta de processos e diretrizes preventivas para a resolução de conflitos); C) Formas de violência e assédio (com as subcategorias: divulgação de rumores no local de trabalho, perseguição, retenção de informação crucial e recusa); e D) Fadiga de compaixão. O fenómeno relaciona-se ao modelo biomédico.

Conclusões: O problema de violência está subjacente a determinantes contextuais organizacionais que não dependem exclusivamente dos profissionais de enfermagem, sendo urgente identificá-los, enfrentá-los e preveni-los.

Palabras chave: Violência no trabalho; enfermagem; cultura organizacional; esgotamento profissional; Chile.

\section{INTRODUCCIÓN}

La violencia es uno de los problemas de mayor impacto en la salud de las personas, por lo que requiere de estrategias de prevención y tratamiento basado en un eficiente afrontamiento․ Chile ocupa el segundo lugar en Latinoamérica en violencia psicológica, en donde las mujeres son las principales víctimas del problema con $69.4 \%$ de denuncias anuales. Sin embargo, en agresiones profesionales el género femenino se reporta como el principal agresor ${ }^{2}$. En este contexto, el entorno laboral de los profesionales de enfermería ha sido considerado un factor de riesgo para sufrir mayor violencia en el sector sanitario3.

En Chile, el estudio realizado por Rodríguez y Paravic 4 en servicios de atención pre hospitalaria, describió un alto nivel de abuso verbal en el lugar de trabajo, tanto en profesionales como técnicos. Los abusadores fueron identificados en $80 \%$ como pacientes y público en general, seguido por $20 \%$ provocado por otros miembros del personal. A nivel global, estudios sobre las causas de violencia en entornos laborales sanitarios han descrito que 61\% se relaciona con aspectos organizacionales, y $17 \%$ con la capacidad de interacción social de las personas involucradas 3 . Esto orienta hacia las posibles causas y soluciones del problema, las que Munir et al..$^{5}$ asociaron en el caso de los profesionales de enfermería a la frustración, resultado de ser poco valorados a nivel social.

De acuerdo a Moss et al. ${ }^{6}$, los profesionales sanitarios tienen cinco veces más posibilidades de sufrir violencia laboral que otras profesiones. Al respecto, en Chile los entornos laborales sanitarios 
demandantes provocan desgaste del personal sanitario, lo que se ha asociado a variados niveles de violencia, aunque falta más evidencia al respecto7. Las enfermeras y estudiantes de enfermería están más expuestas a maltrato psicológico que otras profesiones, lo que se ha descrito como fenómeno en distintos servicios clínicos ${ }^{8-12}$ y afectan con mayor frecuencia a recién egresadas o profesionales menos experimentados ${ }^{13}$. La violencia laboral es una situación no deseable que afecta la dignidad y moral de las enfermeras involucradas, su sentido de valía, su salud física y mental ${ }^{14-15}$.

Laboralmente se convierte en una grave amenaza contra la eficiencia de las organizaciones al dañar el entorno laboral'15, por ello la Organización Internacional del Trabajo (OIT) a partir del Convenio sobre la violencia y acoso laboral del año 2019.16, "proporciona un marco claro para la adopción de medidas, y brinda la posibilidad de forjar un futuro del trabajo sobre la base de la dignidad y el respeto, exento de violencia y acoso", y hace énfasis en abordar las causas subyacentes.

La violencia en enfermería se expresa a menudo como acoso laboral en el que la víctima recibe maltrato, habitualmente psicológico de parte del acosador(a), el cual puede ser vertical u horizontal ${ }^{16-17}$. Este aspecto ha ido tomando mayor importancia, ya que cualquier forma de violencia y acoso contribuye a crear un entorno inseguro y de riesgo para los pacientes, lo que obliga al estamento de enfermería a monitorearlo y subsanarlo6.

Por otra parte, la ausencia de definiciones consensuadas en torno a la violencia laboral dificulta determinar su prevalencia específica, y el reconocimiento del fenómeno en entornos sanitarios ${ }^{18}$. En ese sentido, es un tema emergente, poco estudiado y al cual no se le ha dado la relevancia como problema de salud pública, por lo que se requiere mayor evidencia empírica que devele las características del fenómeno y sus causas ${ }^{17}$.

Se ha planteado que la violencia horizontal podría estar tan arraigada en la cultura de enfermería que limita la posibilidad de reconocerla cuando se presenta o cuando se experimenta, es decir, mientras no se asuma como problema frecuente, será difícil buscar soluciones efectivas ${ }^{19}$.

Dentro de los factores que se han asociado con mayor frecuencia a la violencia laboral se puede mencionar: aumento de profesionales recién egresados, entorno feminizado, estrés asociado a escasez de recursos para el cuidado, clima laboral deficiente, antecedentes de agresiones previas, estilos de liderazgo poco democráticos, y falta de educación cívica ${ }^{6,20-22}$.

En la literatura revisada una de las perspectivas teóricas más utilizadas para la comprensión de la violencia laboral es el modelo de visión cognitiva-fenomenológica-transaccional del estrés y afrontamiento de Lázarus²3, y propuesta para enfermería por Bailey ${ }^{23}$. De acuerdo a Bailey, este modelo es:

a) cognitivo porque "contiene el supuesto de que el pensamiento, la memoria y el significado de los eventos para la persona que los experimenta son los mediadores centrales y los agentes causantes inmediatos en la determinación del estrés y el afrontamiento. Tal valoración es función de la experiencia y la memoria pasadas".

a) fenomenológico porque es la propia valoración individual y cultural que hace la persona, la que se considera el factor crucial en su respuesta.

a) transaccional porque da énfasis a la interacción entre las valoraciones realizadas por el individuo y el entorno en el que se encuentra ${ }^{23}$.

La importancia de este modelo es que, frente a una misma situación o factor de estrés, la forma de afrontamiento de los profesionales de enfermería dependerá de cómo éste perciba la amenaza (evaluación primaria), y evalúe los recursos que tiene para hacer frente o contrarrestar los 
daños (evaluación secundaria). El profesional de enfermería en el modelo es "un sistema dinámico que intenta controlar los niveles de amenaza o demanda a los que está expuesto mediante un afrontamiento eficaz" ${ }^{23}$. Este aspecto "es absolutamente vital para cualquier persona preocupada por su propia salud" ${ }^{23}$ y la de los demás. Asimismo, es fundamental para profesionales de enfermería que se exponen a entornos estresantes y exigentes, ya que manejar variadas estrategias de afrontamiento contribuyen a sobrellevar de la mejor forma estas situaciones, y traspasar este aprendizaje a sus estudiantes y pacientes.

Las actitudes violentas son formas de afrontamiento inadecuadas y se constituyen como un fenómeno complejo y multidimensional que debe ser abordado no sólo por ser destructivo y dañino para el estamento profesional, sino para toda la sociedad, y de no intervenirlo se perpetúan silenciosamenteformas de afrontamiento ineficaz entre profesionales y estudiantes de enfermería ${ }^{24}$. En consecuencia, se propone este estudio con el objetivo de comprender la experiencia de violencia laboral entre enfermeras hospitalarias durante el año 2017 en la ciudad de Valdivia, Chile, lo que puede contribuir a mejorar la comprensión de su génesis y orientar en su solución.

\section{MÉTODO}

La investigación se aborda desde el paradigma interpretativo, metodología cualitativa y análisis de contenido de acuerdo a lo propuesto por Bardin²5, quien lo define como: "un conjunto de instrumentos metodológicos aplicados a contenidos de discursos extremadamente diversificados. Es una hermenéutica controlada, basada en la inferencia". El análisis se basó en la construcción de categorías temáticas abiertas que fueron refinadas en razón de una mayor profundización teórica de los hallazgos.

El estudio se realizó en el Hospital base de Valdivia, se utilizó muestra intencionada y se accedió a los informantes a través de la oficina de gestión de personas, la cual recoge denuncias de maltrato laboral, actuando como intermediario para informar y lograr el consentimiento inicial de los posibles participantes del estudio. También se empleó la técnica de bola de nieve, ya que algunos de los informantes sugirieron entrevistas a otros profesionales que se percibían como violentados.

Se hicieron entrevistas en profundidad a diez profesionales de enfermería, basado en una parrilla de preguntas orientadoras encaminadas a obtener las descripciones de situaciones de violencia experimentadas por los informantes; el significado de estas situaciones; así como los aspectos facilitadores que influyeron en la violencia laboral. Las entrevistas se llevaron a cabo fuera del lugar de trabajo y en condiciones de confort para los sujetos de estudio. Se realizó una entrevista por informante, con una duración promedio de 1.5 horas. En algunos casos se consideró saturada la información, mientras que en otros se privilegió el bienestar evitando procesos de revictimización. Dada la sensibilidad que provoca la temática se contó con un protocolo de derivación psicológico en caso de requerir intervención en crisis con apoyo de experto; sin embargo, nunca se utilizó.

Las entrevistas fueron realizadas por un periodista en calidad de ayudante de investigación, con experiencia en estudios de investigación cualitativa y contención emocional. Esto con el fin de manejar el principal sesgo del estudio, ya que la investigadora principal era funcionaria de la institución en dónde se realizó la investigación.

El perfil deseado para los posibles participantes fue profesionales de enfermería que se percibieran violentados por sus pares y menores de 40 años, pues con base en la literatura, este fenómeno es mucho más frecuente y grave en profesionales jóvenes. De igual forma, tenían 
que cumplir funciones en algunos de los servicios de atención cerrada del hospital y haberse desempeñado en dichos servicios al menos por seis meses. Cabe señalar que no se consideró a internos de enfermería o en etapa final de formación profesional.

La recolección y análisis se llevó a cabo en forma simultánea, emergiendo las primeras categorías para su posterior refinamiento. En vista de que la investigadora principal era iniciada, se realizó una segunda ronda interpretativa en la que participó una asesora y una investigadora externa, lo que llevó a un segundo refinamiento de las categorías e interpretación de los datos. Durante la fase analítica se utilizó el programa ATLAS ${ }^{\circledR}$ TI versión 7.5.4, que permitió organizar el trabajo a distancia y optimizar mejor los tiempos de trabajo de las investigadoras.

Los criterios de rigor ${ }^{26}$ consideraron confiabilidad al levantar datos integramente, realizar transcripción completa y fidedigna, y hacer notas de campo cuando fue posible. Además, se mantuvieron reuniones permanentes entre las investigadoras principales y el ayudante de investigación, con el propósito de orientar de mejor forma el trabajo de campo. Una vez construidas las categorías, se trianguló teóricamente con la investigadora externa para lograr un consenso en la línea teórica que el equipo de investigación consideró relevante para responder al objetivo del estudio.

La investigación se apegó a los criterios éticos ${ }^{26}$, se solicitó la autorización de un Comité de ética de investigación independiente. Se aplicó consentimiento informado a los doce participantes, pero dos desistieron participar.

\section{RESULTADOS}

Participaron en el estudio seis enfermeras y cuatro enfermeros que se desempeñan en servicios de alta demanda del Hospital base de Valdivia, con experiencia laboral entre 1.5 y 9 años, y un promedio de edad de 29.5 años. Del estudio emergen cuatro categorías y ocho subcategorías, las cuales se sustentan en la evidencia empírica encontrada.

A) Percepción de clima organizacional asociada a estilos de liderazgo. La mayoría de los participantes describen una gran cantidad de episodios de violencia psicológica ligados al estilo de liderazgo de sus jefaturas o pares. En este punto los informantes hacen énfasis en los procesos de comunicación y resolución de conflictos, los que reconocen como intrínsecamente asociados al estilo de liderazgo autocrático que se ejerce en el servicio.

- "Las relaciones al final no son muy llevaderas porque hay mucho conflicto de ego, muchos conflictos de interés" (Eg).

- "[...] el ego está siempre ahí, el tratar de desacreditar al otro en vez de potenciarnos como profesionales" (E7).

- "La encargada acá es la que tiene el carácter más fuerte, y ya la conozco hace años, a veces se estresa demasiado y estresa al resto, pero no pasa eso de que si yo me estreso yo la enfrento y nos peleamos. [...], ahora lo que hago es que actúo con ética, el profesionalismo de guardar silencio cuando corresponde" (E2).

- "Fui súper perseguido porque mis colegas me denunciaban porque vine y traje mi licencia médica y me denunciaron porque me vieron acá. "¿Qué tienes que estar haciendo tú con licencia acá?», [...] la situación fue difícil”"(E5). 
No obstante, coinciden en otorgar una mayor valoración positiva hacia el estilo de liderazgo democrático, por sobre estilos autocrátricos o autoritarios, como medio para favorecer un mejor clima organizacional y por tanto, menor violencia.

- "Si tienes una jefatura empática, que sabe escuchar, que se comunica bien, ahí tienes una jefatura que, de perder, no va a perder nunca y va a resolver las cosas de buena manera" (E1).

- "Loconversamos con mijefa y después loconversécon mi colega, haybuena comunicación" (E2).

- "Esta unidad es pequeña, pero con clima amigable, trabajamos tres personas no más y nos comunicamos bien" (E4).

B) Barreras contextuales y actitudinales asociadas a la aparición de conductas violentas. Las barreras contextuales mencionadas por los informantes corresponden a limitaciones presentes en el entorno que les impiden sentirse incluidos en el equipo de enfermería y actuar con eficiencia en la gestión de los cuidados de los pacientes. Las más representativas son:

a) Falta de colaboración profesional:

- "Cuesta mucho que las enfermeras estén siempre unidas, por el tema de los turnos y por el tema de la competitividad, en enfermería se ve harto que son muy competitivas, eso hace dificil remar para el mismo lado (significa cooperar unas con otras)" (E10).

- "Las enfermeras de la parte de hospitalizados nos hicieron una jugada muy fea, [...] les avisaron con un mes de anticipación que ya no íbamos a hacer más esos turnos, ni siquiera nos avisaron"(Eg).

b) Sobrecarga laboral:

- "Tenía mucha carga laboral en cuanto a lo clínico, y lo otro es que mi enfermera jefa también me empezó a dar otras labores, por ejemplo, subrogarla en la jefatura, entonces tuve que empezar a resolver problemas más administrativos que de lo clínico y ahí como que pasé un periodo de mucho estrés" (E2).

- "La pega es harta y estás siempre bajo presión y contra los tiempos para organizar todo rápido" (E7).

- "Ya estoy ansiando las vacaciones, pero ha sido mucha pega, hemos tenido mucho paciente y... hay tratamientos muy largos. Mi horario de trabajo es hasta las cinco de la tarde, pero me estoy yendo tipo siete de la tarde, ocho de la tarde, entonces eso cansa, prácticamente haciendo un turno largo casi todos los dias" (E4).

c) Falta de procesos y pautas preventivas para la resolución de conflictos:

- "Cuando llega una colega nueva está sujeta a todas las miradas, pero... con esa colega, o sea la persona nueva, no somos mala onda, sólo que estamos siempre observando, como pendientes de que no vaya a pasar alguna cosa porque es nueva y no tiene experiencia, [...] no hay mucho tiempo para acompañar" (E6).

C) Formas de violencia o acoso vivido. Los participantes se refieren a diferentes expresiones de violencia o acoso experimentado en el entorno laboral.

a) Difundir rumores laborales: los participantes refieren haber sido objeto o testigos de rumores, que se transmiten de un profesional a otro, con la finalidad de desacreditar a un par: 
"Esa enfermera se encargó de emitir comentarios del problema vivido a otro servicio donde estaba trabajando, tenemos comentarios de que la enfermera es muy competitiva y confrontacional, y en el servicio no estamos acostumbrados a eso" [E10].

b) Persecución: los participantes mencionan haber sido objeto de búsqueda activa de errores en sus acciones, con el fin de ser perjudicadas laboralmente. Refieren que esto ocurre con mayor frecuencia de forma horizontal, es decir, de profesionales con mayor experiencia hacia novatos:

"Las colegas entre ellas son muy competitivas, cuando pueden te perjudican, repitiéndose el ciclo. La enfermera que fue nueva supervisa a la más nueva y hurga hasta encontrar algo deficiente y la perjudica" [E8].

"Les pregunto de todo, esto hace que me tengan mucho miedo". [E6]

c) Humillación pública: los participantes se refieren al menoscabo que sienten cuando se les llama la atención frente a otros, ya sea para reprender o cuestionar sus capacidades públicamente:

"Una vez me llamó la atención una colega frente al público. Fue fuerte porque me llamó la atención frente a otra colega y me dijo que, si yo no estaba capacitada para seguir trabajando en este servicio, que mejor me fuera de aqui" [E5].

d) Retener información crucial: los participantes mencionan que se les niega u oculta información crucial para realizar el trabajo, lo que deja al profesional de enfermería vulnerable a cometer errores:

"Ella realiza procedimientos en un pabellón y se ausentó, por lo que debí cubrir, pero yo desconocía las acciones y me dejó sólo anotado lo que se debía realizar, pero no me permitió hablar con ella, yo estaba súper estresada" [E8].

e) Desautorización: los participantes mencionan que se le resta valor profesional a sus opiniones, sugerencias o trabajo:

"Ella era súper rígida, si yo no realizaba el trabajo como ella decía, estaba mal hecho. Era prepotente conmigo" [E4].

"Yo me sentí pasada a llevar, ella revisaba mi trabajo para comprobar si lo expresado en entrega de turno era verdadero" [E9].

D) Fatiga de compasión. Los participantes refieren que, con un clima laboral hostil se deteriora el trato compasivo hacia los pacientes, y se deshumanizan los cuidados:

- "Los mismos pacientes a veces reclaman porque les da susto, la gente se siente como intimidada al lado de ella, prefieren que los atienda otra persona" (E6).

- "[...] súper asustada porque era así, creo que una vez a un compañero hasta le dijo un garabato, era súper despectiva y si te podía retar lo hacía, haciéndote sentir muy humillada delante del paciente" (E10).

\section{DISCUSIÓN}

\section{A) Percepción de clima organizacional asociada a estilo de liderazgo}

La violencia laboral que experimentaron los informantes está asociada a un clima organizacional deficiente, relacionado con distintos estilos de liderazgo. El clima organizacional corresponde a una 
dimensión esencial en el éxito de las organizaciones, ya que permite crear entornos amables en que los profesionales pueden tener la certeza de que podrán contar con apoyo para lograr su máximo desarrollo, incluyendo el manejo de situaciones de estrés. Este aspecto influye en la moral y actitud de las personas, generando motivación y compromiso con otros miembros de la organización ${ }^{27}$. Con respecto a este estudio, los informantes relacionaron el deterioro del clima organizacional con el liderazgo autocrático ejercido por algunas jefaturas y pares, lo que influye en el deterioro de la comunicación y confianza, genera un clima hostil que poco a poco lleva al desgaste y afecta la eficiencia de los cuidados.

En otro sentido, los informantes valoran positivamente los liderazgos más democráticos y transformacionales, incorporan la idea de una jefatura horizontal, con buena comunicación, que motive y que encarne valores fundamentales de la profesión de enfermería. En este punto se reconoce que el líder de una organización debe ser competente, capaz de "motivar y alentar al personal para promover la realización del trabajo y lograr los objetivos propuestos" ${ }^{28}$. Por el contrario, en el caso de líderes autocráticos se ha reportado en la literatura que dificultan realizar un adecuado trabajo en equipo, afectando negativamente el entorno de cuidado y a los pacientes ${ }^{27-29}$, lo que coincide con lo expuesto por los participantes.

El estudio realizado por Bustamante et $a 1 .^{30}$ en Chile sobre clima organizacional en hospitales auto gestionados concluyó que un clima organizacional deteriorado provoca que "los funcionarios autorregularan en forma defensiva sus actitudes, conductas y capacidad de colaboración al sistema organizacional, lo que dificulta su propia forma de trabajo, la toma de decisiones y la capacidad de adaptación". Por lo tanto, no atender estas situaciones en forma oportuna, especialmente en los entornos clínicos, favorecería círculos viciosos, en donde se perpetúan conductas y actitudes desadaptadas y violentas. Desde esta perspectiva, construir un buen clima laboral y mantenerlo contribuye al desarrollo de una cultura organizacional sana, aspecto clave para lograr un cuidado eficiente y seguro para los pacientes ${ }^{6}$.

\section{B) Barreras contextuales y actitudinales asociadas a la aparición de conductas violentas}

Las barreras contextuales o actitudinales responden a la dimensión transaccional del modelo de Lázarus $^{23}$ y corresponden a cualquier factor que los profesionales de enfermería reconozcan como fuente de estrés externa, provengan éstas del entorno físico o de actitudes que sean percibidas como estresantes y que originan un afrontamiento ineficiente. La identificación de estas brechas puede contribuir a corregir el problema, previniendo riesgos que puedan dañar la salud de este personal y de los pacientes. Sin embargo, los factores contextuales no siempre dependen de los profesionales de enfermería, más bien están determinados por factores estructurales, relacionados con políticas sanitarias y decisiones gerenciales de los hospitales ${ }^{31-32}$.

La diferencia con las barreras actitudinales, es que, en estas últimas, puede intervenirse en el corto plazo y por los mismos profesionales de enfermería. En la evaluación primaria se debe partir de reconocer la existencia de un conflicto de difícil manejo, para luego realizar un análisis sobre las capacidades tendientes a subsanarlo, lo que corresponde a la evaluación secundaria del modelo. Las actitudes profesionales responden a una dimensión personal (psicológica-moral) y una social (profesional), las que no siempre reciben la atención necesaria ${ }^{14}$, debido a que en los entornos clínicos se ha privilegiado el desarrollo de competencias técnicas y procedimentales, en correspondencia a la hegemonía del modelo biomédico ${ }^{31}$. En el presente estudio se identificaron cuatro dimensiones asociadas a esta categoría: 
a) Falta de colaboración profesional

La colaboración profesional según Emich33 "es un proceso interprofesional mediante el cual las enfermeras se unen, y forman un equipo para resolver un problema de atención al paciente, o del sistema de salud con los miembros del equipo, se comparte conocimiento y recursos de forma respetuosa". No obstante, se han descrito diferentes barreras para lograr un entorno colaborativo en enfermería, las cuales coinciden con hallazgos de este estudio. Se ha evidenciado que la presencia de discursos críticos y poco constructivos, ya sea de jefaturas o pares, provoca recelo entre los profesionales, quienes prefieren abstenerse de proponer nuevas ideas, lo que limita la innovación, la participación, deteriora la comunicación, y colaboración con otros miembros del equipo ${ }^{30}$.

b) Sobrecarga laboral

La revisión realizada por Canales et al.10 describió que en Chile las condiciones laborales de los profesionales de enfermería se caracterizan por la precariedad, demanda laboral y limitados recursos. Se describen, además, problemas de estrés, fatiga y burnout. La falta de control de enfermería sobre los recursos necesarios para realizar un cuidado eficiente y el gran flujo de trabajo califican al entorno de trabajo de la profesión como extremadamente estresante $4,6-8,1,1,14,22$. Este aspecto corresponde a una barrera contextual que afecta la salud mental de los profesionales de enfermería, quienes a pesar de experimentar condiciones de trabajo adversas y sobre exigidas, deben velar por el bienestar de los pacientes ${ }^{6,22}$. En este punto el estudio concuerda con estos aspectos, lo que sería una de las principales causas de estrés, de desgaste y de deterioro del clima organizacional que propicia conductas violentas entre las enfermeras ${ }^{31}$.

c) Falta de procesos y pautas preventivas para la resolución de conflictos

A nivel profesional, estudios sobre violencia en estudiantes de las carreras sanitarias han demostrado que este problema se inicia en etapas muy tempranas de la formación profesional ${ }^{9}$. Esto último debe ser motivo de preocupación permanente del estamento profesional dada la creciente denuncia de violencia y acoso en los servicios sanitarios, la que, en gran parte, según mencionan Rodríguez y Paravic ${ }^{4}$, proviene de los usuarios y público en general, pero también desde el mismo equipo de trabajo.

Chile ha sido descrito como un país con alta prevalencia de estrés laboral, lo que se asocia a altos costos para el sistema. En general, las enfermeras no reconocen las conductas relacionadas con la violencia cuando las presencian o las experimentan, lo cual ha limitado la búsqueda de soluciones y podría relacionarse con la naturalización del estrés y ritualización de estas conductas ${ }^{19}$. Así, cuando existe violencia, se atribuyen dichas conductas a la personalidad de algunos de los profesionales involucrados, sin que se maneje como parte de un problema de la organización y se aborde integralmente. Aunado a ello, existen estudios que describen el tipo de afrontamiento al estrés por parte del profesional de enfermería, donde lo definen como sublimador, lo que refleja posiblemente una actitud aprendida en la cual se opta por la evitación de conflictos ${ }^{14}$.

De acuerdo al estudio realizado por Babaei y Taleghani ${ }^{32}$ sobre violencia en servicios de enfermería, se reconoce un fuerte componente organizacional que no se subsana con simples capacitaciones o intervenciones ocasionales, sino con cambios profundos asociados más bien al modelo sanitario biomédico. Desde otra perspectiva, Crawford et al. ${ }^{22}$ proponen desarrollar pautas éticas dirigidas a fortalecer la educación democrática y cívica para la profesión de enfermería, pues los espacios laborales debiesen aportar a la construcción de una sociedad sana, 
democrática y en paz. La autora sugiere que el desarrollo de competencias en ética cívica es una valiosa herramienta para desarrollar habilidades para la vida con otros; sin embargo, el estamento de enfermería aún no las incorpora en su formación, a pesar de la importancia para desarrollar el rol sociopolítico de la profesión.

\section{C) Formas de violencia y acoso horizontal}

La violencia horizontal en enfermería se define como el comportamiento agresivo y dañino por parte de un profesional de enfermería o un grupo de profesionales hacia otros compañeros y compañeras. Este incluye no sólo agresiones verbales o físicas, sino falta de reconocimiento a sus acciones, palabras y otros comportamientos que menoscaban su dignidad ${ }^{19}$. El convenio de la OIT ${ }^{16}$ con los Estados miembros ha contribuido a visibilizar el problema de la violencia y acoso laboral a nivel global, recomendando a los países "adoptar medidas para que el trabajo se realice sobre la base de un trato digno y de respeto".

El primer esfuerzo para cambiar comportamientos violentos y de acoso es reconocer la existencia del problema, para luego desarrollar nuevas prácticas que reflejen un entorno colaborativo ${ }^{6}$. Los hallazgos del estudio describen las siguientes formas de violencia y acoso laboral sufrida por los informantes: difundir rumores laborales, persecución, humillación pública, retener información crucial y desautorización. Estas malas prácticas son indicadores de un clima organizacional deteriorado, dentro del cual la comunicación formal es deficiente, poco democrática y el respeto por los derechos de las personas son vulnerados.

$\mathrm{Al}$ respecto, estudios coinciden que el rumor intencionadamente negativo afecta de manera relevante el entorno clínico de enfermería, deteriora la calidad del cuidado, menoscaba a las personas y desgasta a los profesionales involucrados ${ }^{34}$. La revisión de la literatura realizada por Bambi et al. ${ }^{11}$, que incluyó 25 artículos a nivel global, describió que al igual que los hallazgos de este estudio, los profesionales jóvenes se sienten acosados por los más experimentados. Esto se atribuye a la falta de experiencia, menos confianza en su trabajo y desconocimiento de las reglas institucionales. Por su parte, en el caso de los profesionales con más experiencia y que acosan a los más jóvenes se reportó una personalidad narcisista, baja autoestima y un deficiente liderazgo.

\section{D) Fatiga de compasión}

La compasión es un valor fundamental del cuidado y un componente necesario para brindar servicios de enfermería de calidad. Sus dimensiones son habitualmente mencionadas por los pacientes, estudiantes y profesionales de enfermería como elementos críticos de la humanización de los cuidados y los derechos humanos fundamentales: empatía, respeto, y trato digno. Estudios a nivel global dan cuenta que un clima organizacional deficiente produce desgaste psicológico en los profesionales de enfermería con la aparición de fatiga de compasión y pérdida de la resiliencia35.

Imponer deberes de cuidado a las enfermeras en un clima organizacional orientado a tareas, con solicitudes más allá de lo posible, provocaría que las enfermeras no presten atención a todas las necesidades de sus pacientes, y el cuidado sea menos compasivo ${ }^{32}$. Esta situación concuerda con lo encontrado en este estudio, en el que los profesionales de enfermería mencionan que una sobrecarga de trabajo y un clima organizacional deteriorado y falto de colaboración intergeneracional tiene consecuencias en lo personal al generar agotamiento crónico, ocupar tiempo en solucionar problemas del entorno, y limita mantener la relación de ayuda y compasiva con los pacientes. 


\section{CONCLUSIONES}

Las experiencias de violencia que mencionan los profesionales de enfermería dan cuenta de las categorías: A) Percepción de clima organizacional asociado a estilos de liderazgo; B) Barreras contextuales y actitudinales asociadas a la aparición de conductas violentas (con las subcategorías: falta de colaboración, sobrecarga laboral, falta de procesos y pautas preventivas para la resolución de conflictos); C) Formas de violencia y acoso (con las subcategorías: difundir rumores laborales, persecución, retener información crucial y desautorización); y D) Fatiga de compasión. Estas se asocian a formas de estrés organizacional mencionados en una emergente literatura al respecto. Consideramos que la raíz del problema es la hegemonía del modelo biomédico con sus ya conocidas limitaciones y la falta de modelos de enfermería de naturaleza holística en la práctica clínica.

Como estrategia de intervención, el modelo de estrés y afrontamiento de Lázarus ofrece la posibilidad detener una mayor comprensión delas consecuencias delfenómenoy generarestrategias de afrontamiento eficientes, diseñar pautas para el manejo del conflicto en la organización, y prevenir tempranamente la fatiga de compasión y conductas violentas que debilitan la eficiencia de los cuidados.

Finalmente, sugerimos profundizar en la fatiga de compasión, la cual visibiliza los efectos de la violencia laboral en la salud psicológica de los profesionales y el daño a la eficiencia de los cuidados. Cabe señalar que la principal limitación del estudio han sido los sesgos en las muestras al no considerar mayor variabilidad (muestras de diversas edades, experiencia laboral, y proporcionalidad en el género), lo que será abordado en estudios posteriores.

\section{RESPONSABILIDADES ÉTICAS}

Protección a personas y animales. En este estudio no se realizaron experimentos con personas o animales.

Confidencialidad. Se garantizó la confidencialidad y anonimato de las personas participantes.

Conflicto de intereses. Los autores declaran no tener conflicto de intereses.

Financiamiento. Ninguno.

\section{REFERENCIAS}

1. Organización Mundial de la Salud (OMS). Informe mundial sobre la violencia y la salud Ginebra: OMS; 2002. https://bit.ly/3fr7Np4

2. Díaz-Berr X, Mauro-Cardarelli A, Toro-Cifuentes JP, Villarroel-Poblete C, Campos- Schwarze D. Validación del Inventario de Violencia y Acoso Psicológico en el Trabajo -IVAPT-PANDO- en tres ámbitos laborales chilenos. Cienc. Trab. 2015; 17(52): 7-14. https://bit.ly/3cyplxt

3. Palma A, Ansoleaga E, Ahumada M. Violencia laboral en trabajadores del sector salud: revisión sistemática. Rev. méd. Chile. 2018; 146(2):213-22.

http://dx.doi.org/10.4067/s0034-98872018000200213

4. Rodríguez-Campo V, Paravic-Klijn T. Abuso verbal y acoso laboral en servicios de atención prehospitalaria en el Chile. 2017; 25: 1-8. http://dx.doi.org/10.159o/1518-8345.2073.2956

5. Munir Y, Ghafoor MM, Rasli AM. Exploring the relationship of horizontal violence, organizational cynicism and turnover intention in the context of social exchange theory. Int J Hum Rights Healthc. 2016; 9(4): 254-66. https://doi.org/10.1108/IJHRH-08-2016-0014 
6. Moss E, Seifert PC, O'Sullivan A. Registered nurses as interprofessional collaborative partners: creating value-based outcomes. Online J Issues Nurs. 2016; 21(3): 4. https://cutt.ly/xnPdFr8

7. Jiménez RE, Bachelet VC, Gomolán P, Lefio LÁ, Goyenechea M. Violence and burnout in health care emergency workers in Santiago, Chile: A survey-based cross-sectional study. Int Emerg Nurs. 2019; 47: 1-8. https://doi.org/10.1016/j.ienj.2019.100792

8. Jiménez-Paneque R, Pavés-Carvajal JR Enfermedades y riesgos laborales en trabajadores de servicios de urgencia: revisión de la literatura y acercamiento a Chile. Medwave. 2015; 15(7): 1-9.

https://doi.org/10.5867/medwave.2015.07.6239

9. Uribe MA, Illesca M. Burnout en estudiantes de enfermería de una universidad privada. Inv Ed Med. 2017; 6(24): 234-41. https://doi.org/10.1016/j.riem.2016.11.005

10. Canales-Vergara M, Valenzuela-Suazo S, Paravic-Klijn T. Condiciones de trabajo de los profesionales de enfermería en Chile. Enferm. univ. 2016;13(3): 178-86.

http://dx.doi.org/10.1016/j.reu.2016.05.004

11. Bambi S, Foà C, De Felippis C, Lucchini A, Guazzini A, Rasero L. Workplace incivility, lateral violence and bullying among nurses. A review about their prevalence and related factors. Acta Bio Med. 2018; 89(S-6): 51-79. https://doi.org/10.23750/abm.v89i6-S.7461

12. Longo J. Combating disruptive behaviors: Strategies to promote a healthy work environment. Online J Issues Nurs. 2010; 15(1). Manuscrito 5. https://ng.cl/qnsjj

13. Martinic-Gutiérrez KP, Souza-Ramos FR, De Lima-Dalmolin G. Síndrome de Burnout en profesionales de enfermería de la ciudad de Punta Arenas, Chile. Texto contexto-enferm. 2020; 29(spe): 1-12. https://doi.org/10.1590/1980-265x-tce-2019-0273

14. Vargas-Celis I, Concha-Méndez CA. Angustia moral, señal de problemas éticos en la práctica de Enfermería oncológica: revision de literatura. Aquichan. 2019; 19(1):1-16.

https://doi.org/10.5294/aqui.2019.19.1.3

15. Sharma RK, Sharma V. Work place violence in nursing. J Nurs Care. 2016; 5(2): 1-3. https://doi.org/10.4172/2167-1168.1000335

16. Superintendencia de seguridad social (SUSESO). OIT: Convenio sobre la violencia y el acoso, 2019 Cinco preguntas clave. Chile: SUSESO; 2019. https://bit.ly/31scsyI

17. Rodríguez VA, Paravic TM. Un modelo para investigar violencia laboral en el sector salud. Rev. Gaúcha Enferm. 2013; 34(1): 196-200. https://doi.org/10.1590/S1983-14472013000100025

18. Crawshaw L. Workplace bullying? Mobbing? Harassment? Distraction by a thousand definitions. Consult Psychol J. 2009; 61(3): 263-7. https://doi.org/10.1037/a0016590

19. Taylor R. Nurses' perceptions of horizontal violence. Glob Qual Nurs Res. 2016; 3: 1-9. https://doi.org/10.1177/2333393616641002

20. Mohamed HA, Higazee MZA, Goda S. Organizational justice and workplace bullying: The experience of nurses. Am J Nurs Res. 2018; 6(4): 208-13. https://ng.cl/fu31

21. Wei CY, Chiou ST, Chien LY, Huang N. Workplace violence against nurses-prevalence and association with hospital organizational characteristics and health-promotion efforts: Cross-sectional study. Int J Nurs Stud. 2016; 56: 63-70. https://doi.org/10.1016/j.ijnurstu.2015.12.012

22. Crawford CL, Chu F, Judson LH, Cuenca E, Jadalla AA, Tze-Polo L, et al. An integrative review of nurse-to-nurse incivility, hostility, and workplace violence: A GPS for nurse leaders. Nurs Adm Q. 2019; 43(2): 138-56. https://ng.cl/dhzar

23. Bailey R, Clarke M. Stress and coping in nursing. Singapore: Chapman and Hall; 1989. https://doi.org/10.1007/978-1-4899-2941-9 
24. Seguel F, Valenzuela S. Relación entre la fatiga laboral y el síndrome Burnout en personal de enfermería de centros hospitalarios. Enferm. univ. 2014; 11(4): 119-27.

https://doi.org/10.1016/S1665-7063(14)70923-6

25. Bardin L. Análisis de contenido. 2da ed. Madrid: Akal Universitaria; 1996.

26. Noreña AL, Alcaraz-Moreno N, Rojas JG, Rebolledo-Malpica D. Aplicabilidad de los criterios de rigor y éticos en la investigación cualitativa. Aquichan 2012; 12(3): 263-74.

https://doi.org/10.5294/aqui.2012.12.3.5

27. Safan SM, Diab GM, Rashad RM. The relationship between organizational citizenship behavior and organizational climate among nursing staff. Int J Nur Res. 2018; 4(4): 254-61.

https://ng.cl/tw4wi

28. Kamal-Ahmed A, Abdeldayem-Ata A, Abd-Elhamid ZN. Relationship between the leadership behaviors, organizational climate, and innovative work behavior among nurses. Am J Nurs Res. 2019; 7(5): 870-8. https://ng.cl/t2s8c

29. Pérez-Fuentes MC, Gázquez JJ, Molero MM, Oropesa NF, Martos A. Violence and job satisfaction of nurses: Importance of a support network in healthcare. Eur. J. Psychol. Appl. to Leg. Context. 2021;13(1): 21-8. http://dx.doi.org/10.5093/ejpalc2021a3

30. Bustamante-Ubilla MA; Grandón-Avendaño ML, Lapo-Maza MC. Caracterización del clima organizacional en hospitales de alta complejidad en Chile. Estud. Gerenc. 2015; 31(137): 432-40. https://doi.org/10.1016/j.estger.2015.08.003

31. Galián-Muñoz I, Ruiz-Hernández JA, Llor-Esteban B, López-García C. User violence and nursing staff burnout: The modulating role of job satisfaction. J Interpers Violence. 2016; 31(2): 302-15. https://doi.org/10.1177/0886260514555367

32. Babaei S, Taleghani F. Compassionate care challenges and barriers in clinical nurses: A qualitative study. Iran J Nurs Midwifery Res. 2019; 24(3): 213-9. https://doi.org/10.4103/ijnmr.IJNMR_100_18

33. Emich C. Conceptualizing collaboration in nursing. Nurs Forum. 2018; 53(4): 567-73. https://doi.org/10.1111/nuf.12287

34. Georganta K, Panagopoulou E, Montgomery A. Talking behind their backs: Negative gossip and burnout in Hospitals. Burn Res. 2014; 1(2): 76-81. https://doi.org/10.1016/j.burn.2014.07.003

35. Peters E. Compassion fatigue in nursing: A concept analysis. Nurs Forum. 2018; 53(4): 466-80. https://doi.org/10.1111/nuf.12274 